\title{
Polaron Effects on Nonlinear Optical Properties of a Hydrogenic Impurity in a CdTe/ZnTe Quantum Dot
}

\author{
A. Azhagu Parvathi ${ }^{a}$ And A. John Peter ${ }^{b, *}$ \\ ${ }^{a}$ Dept. of Physics, VV Vanniaperumal College for Women, Virudhunagar-626 001, India \\ ${ }^{b}$ Dept. of Physics, Govt. Arts College, Melur-625 106, Madurai, India
}

(Received January 17, 2012; in final form July 24, 2013)

\begin{abstract}
Hydrogenic donor impurity binding energy is obtained in a $\mathrm{Zn}_{x} \mathrm{Cd}_{1-x} \mathrm{Te} / \mathrm{ZnTe}$ strained quantum dot taking into account the phonon confinement effect. The interaction of the electron and the phonon modes are expressed in terms of the Fröhlich interaction Hamiltonian. The binding energy is obtained for various $\mathrm{Zn}$ composition using the Aldrich-Bajaj effective potential. Calculations have been obtained using the Bessel function as an orthonormal basis for different confinement potentials of barrier height considering the internal electric field induced by the spontaneous and piezoelectric polarizations. Polaron induced linear and third-order nonlinear optical absorption coefficients and the changes of refractive index as a function of incident photon energy are observed. Our results coincide with the recent observations of a hydrogenic impurity binding energy in a CdTe/ZnTe quantum dot solved analytically. It is observed that the potential taking into account the effects of phonon makes the hydrogenic binding energies larger than the obtained results using a Coulomb potential screened by a static dielectric constant and the optical properties of hydrogenic impurity in a quantum dot are strongly affected by the confining potential and the quantum size. It is found that the geometry of the quantum dot, zinc concentration and the effect of phonon have a great influence on the absorption coefficient and refractive index changes of the dot. It is also observed that the magnitude of the absorption coefficients enhances with the inclusion of phonon effect.
\end{abstract}

DOI: 10.12693/APhysPolA.124.706

PACS: 73.21.Fg, 42.65.An, 71.38.-k

\section{Introduction}

Nonlinear optical properties in low dimensional semiconductor systems such as quantum wells, quantum well wires, and quantum dots are of considerable interest due to their potential applications in device fabrications; further they can be easily fabricated with the latest and sophisticated techniques [1-3]. Among the nonlinear optical properties, the absorption coefficients, changes of refractive index and the interband emission energy are given due attention in quantum dots $[4,5]$. Sahin [6] investigated the nonlinear optical properties of a one- and two-electron systems in a spherical quantum dot with and without a hydrogenic donor impurity. Very recently, some optical nonlinear properties such as the absorption coefficients and refractive index changes of a hydrogenic impurity in an ellipsoidal quantum dot have been investigated, using the compact-density matrix formalism and an iterative method [7].

Wide band gap II-VI semiconductor nanostructure materials have been used for practical applications of optoelectronic devices nowadays operating in the visible region [8]. Among these II-VI semiconductors, CdTe/ZnTe systems have attracted much attention for optoelectronic devices with the short wavelength ranges [9] and as devices for spin transfer [10-12]. Interband transitions in $\mathrm{CdTe} / \mathrm{ZnTe}$ square quantum wells have been investigated

*corresponding author; e-mail: a.john.peter@gmail.com and determined from optical gain spectra at different temperatures by varying well width of CdTe [13]. With the recent advanced technology, the electron-phonon interaction in quantum dots plays an important role in polar crystals [14]. The interaction of electrons with phonons constitute a quasi-particle so called as bound polaron, ultimately its effective mass increases in a polar semiconductor. The recent results show that these interactions are more important in electronic and optical properties when the dimensions are reduced $[15,16]$. This reduction in dimensionality shows the quantum size effects on optoelectronic properties. The phonon confinement effect of the Mot-Wannier exciton binding energy in a quantum wire has been discussed [17]. Confinement of carriers in quantum dots guides the creation of discrete energy levels. Eventually, the size, shape and the confinement potential barrier will considerably change the physical properties. The binding energies of excitons in quantum well structures in the influence of uniform electric field have been investigated by taking into account the exciton longitudinal optical phonon interaction [18].

In the present paper, the hydrogenic impurity binding energy is investigated in a $\mathrm{CdTe} / \mathrm{ZnTe}$ polar quantum dot nanostructure. The interaction of the electron and the phonon modes are expressed in terms of the Fröhlich interaction Hamiltonian. Some optical properties of hydrogenic impurity in a strained $\mathrm{Zn}_{x} \mathrm{Cd}_{1-x} \mathrm{Te} / \mathrm{ZnTe}$ quantum dot with and without the inclusion phonon assuming a spherically confinement potential for various $\mathrm{Zn}$ concentration have been discussed. Variation of donor binding energy as a function of dot radius for various $\mathrm{Zn}$ 
content has been performed using single band effective mass approximation. Calculations have been obtained using the Bessel function as an orthonormal basis for different confinement potentials of barrier height considering the internal electric field induced by the spontaneous and piezoelectric polarizations. In Sect. 2, the theoretical model used in our calculations of obtained eigenfunctions and eigenenergies of electron states, the linear and non-linear optical absorption coefficients and the refractive index changes have been discussed. The results and discussion are presented in Sect. 3. A brief summary and results are presented in the last section.

\section{Model and calculations}

\subsection{Polaron induced binding energy}

We consider a hydrogenic impurity located at the centre of a $\mathrm{Zn}_{x} \mathrm{Cd}_{1-x}$ Te spherical dot confined by a spherical potential well (ZnTe). The confining potential is assumed to be zero inside and $V$ outside. Within the framework of single band effective mass approximation, the Fröhlich interaction Hamiltonian of the system, a strained $\mathrm{Zn}_{x} \mathrm{Cd}_{1-x} \mathrm{Se} / \mathrm{ZnSe}$ quantum dot with the radius $R$, can be written as

$$
H=H_{\mathrm{e}}+H_{\mathrm{ph}}+H_{\mathrm{e}-\mathrm{ph}},
$$

where

$$
H_{\mathrm{e}}=-\frac{\hbar^{2}}{2 m_{\mathrm{ep}}^{*}} \nabla^{2}+V_{\mathrm{AB}}(r)+V(r),
$$

where $V(r)$ is the strain induced confinement potential of the particle and $V_{\mathrm{AB}}(r)$ is the effective Coulomb potential as given below. The strain induced confinement potential can be written as a sum of energy band offsets of the conduction band and the static electric potential induced by the built-in electric field.

The strength of the built-in electric field $F$ caused by the spontaneous and piezoelectric polarizations in the $\mathrm{Zn}_{x} \mathrm{Cd}_{1-x}$ Te strained quantum dot expressed as [19]:

$$
F=\left\{\begin{array}{cc}
\left|\frac{P_{\mathrm{SP}}^{\mathrm{ZnCdTe}}+P_{\mathrm{PE}}^{\mathrm{ZnCdTe}}-P_{\mathrm{SP}}^{\mathrm{ZnTe}}}{\varepsilon_{0} \varepsilon_{\mathrm{e}}^{\mathrm{ZnCdTe}}}\right|, & r<R, \\
0, & r \geq R .
\end{array}\right.
$$

Here, $\varepsilon_{\mathrm{e}}^{\mathrm{ZnCdTe}}$ is the electronic dielectric constant of $\mathrm{ZnCdTe}$ and $P_{\mathrm{PE}}^{\mathrm{ZnCdTe}}, P_{\mathrm{SP}}^{\mathrm{ZnCdTe}}$ and $P_{\mathrm{SP}}^{\mathrm{ZnTe}}$ are the piezoelectric polarizations and spontaneous polarizations of $\mathrm{ZnCdTe}$ and the spontaneous polarization of $\mathrm{ZnTe}$, respectively. The above values can be generally calculated by the polarity of the crystal and the strains of the quantum nanostructure. Since the wurtzite crystal lattice of $\mathrm{ZnCdTe}$ and $\mathrm{ZnTe}$ lack inversion symmetry, the heterostructure will have spontaneous polarization $\left(P^{\mathrm{SP}}\right)$ and the piezoelectric polarization $\left(P^{\mathrm{PZ}}\right)$ due to strain caused by the lattice mismatch between CdTe and ZnTe material. by

The piezoelectric polarization along the $c$-axis is given$$
P^{\mathrm{PZ}}=e_{31}\left(\varepsilon_{x x}+\varepsilon_{y y}\right)+e_{33} \varepsilon_{z z}
$$

with $\varepsilon_{x x}=\varepsilon_{y y}=\frac{a(\mathrm{ZnTe})-a(\mathrm{ZnCdTe})}{a(\mathrm{ZnTe})}$ and $\varepsilon_{z z}=-2 \frac{C_{13}}{C_{33}} \varepsilon_{x x}$.
The piezoelectric polarization is given by

$$
P^{\mathrm{PZ}}=2 \varepsilon_{x x}\left(e_{31}-\varepsilon_{33} \frac{C_{13}}{C_{33}}\right) .
$$

Thus the total polarization is given by

$$
\boldsymbol{P}=\boldsymbol{P}^{\mathrm{PZ}}+\boldsymbol{P}^{\mathrm{SP}} \text {. }
$$

The band gap of the material is given by [20]:

$$
E_{\mathrm{g}}\left(\mathrm{Zn}_{x} \mathrm{Cd}_{1-x} \mathrm{Te}\right)=1.51+0.45 x+0.31 x^{2}[\mathrm{eV}] .
$$

The above expression of band gap has been obtained by varying $\mathrm{Zn}$ concentration in $\mathrm{ZnCdTe/ZnTe} \mathrm{quantum}$ dot (inset in Fig. 1). The quadratic term in Eq. (7) is correlated with the bowing of optical band gap.

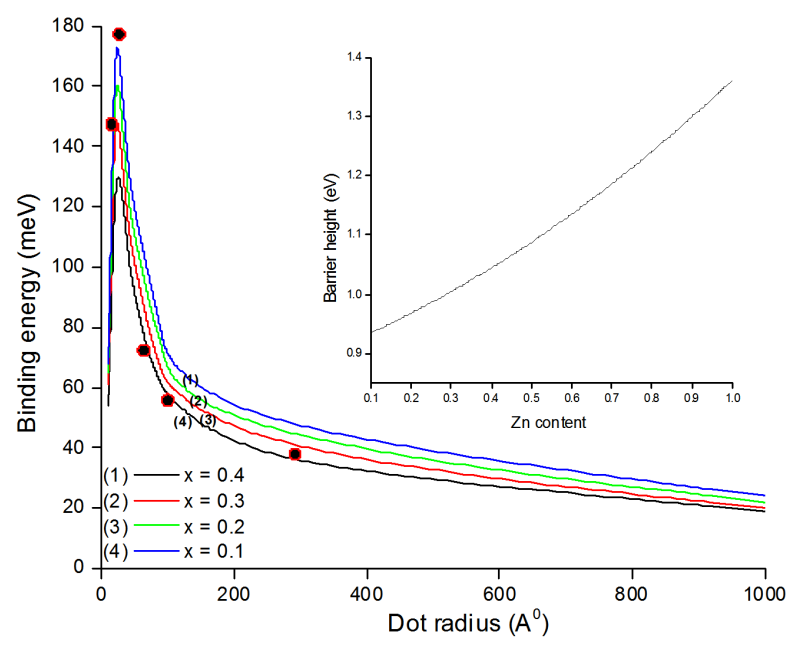

Fig. 1. Variation of binding energy as a function of dot radius for a $\mathrm{Cd}_{1-x} \mathrm{Zn}_{x} \mathrm{Te} / \mathrm{ZnTe}$ quantum dot for various $\mathrm{Zn}$ content; the inset shows the variation of barrier height with the $\mathrm{Zn}$ concentration.

The electron-polaron mass is given by

$$
m_{\mathrm{ep}}^{*}=m_{j}^{*}(1+\alpha / 12)(1-\alpha / 12) \text {. }
$$

The electron effective mass, $m_{j}^{*}$, in the heterosystem, is given by

$$
m_{j}^{*}= \begin{cases}m_{\mathrm{I}}^{*}, & r<R \\ m_{\mathrm{II}}^{*}, & r \geq R .\end{cases}
$$

$m_{\mathrm{I}}^{*}$ and $m_{\mathrm{II}}^{*}$ denote the effective mass of the inside $(\mathrm{CdTe})$ and outside the quantum dot ( $\mathrm{ZnTe})$.

The phonon part of Eq. (1) is given by

$$
H_{\mathrm{ph}}=\sum_{q} \hbar \omega_{\mathrm{LO}} a_{q}^{+} a_{q}
$$

and the electron-LO phonon interaction $\left(H_{\mathrm{e}-\mathrm{ph}}\right)$ is given by $[7,21]$ :

$$
H_{\mathrm{e}-\mathrm{ph}}=\sum_{q}\left(V_{q} \mathrm{e}^{\mathrm{i} q r} a_{q}+V_{q}^{*} \mathrm{e}^{-\mathrm{i} q r} a_{q}^{+}\right),
$$

where

$$
V_{q}=-\frac{\mathrm{i} \hbar \omega_{\mathrm{LO}}}{q}\left(\frac{4 \pi \alpha_{\mathrm{e}}}{\Omega}\right)^{1 / 2}\left(\frac{\hbar}{2 m_{\mathrm{ep}}^{*} \omega_{\mathrm{LO}}}\right)^{1 / 4}
$$

with 


$$
\alpha_{\mathrm{e}}=\frac{e^{2}}{2 \hbar \omega_{\mathrm{LO}}}\left(\frac{2 m_{\mathrm{ep}}^{*} \omega_{\mathrm{LO}}}{\hbar}\right)^{1 / 2}\left(\frac{1}{\varepsilon_{\infty}}-\frac{1}{\varepsilon_{0}}\right),
$$

where $a_{q}^{+}$and $a_{q}$ are the creation and annihilation operators for the LO phonon, respectively. $\hbar \omega_{\mathrm{LO}}$ is the energy of the optical phonon. $\alpha_{\mathrm{e}}$ is the electron-phonon coupling constant which depends on the material parameter taken into account. $\Omega$ is the volume of the quantum dot. $\varepsilon_{0}$ and $\varepsilon_{\infty}$ are the static and optical dielectric constant.

Then the eigenfunctions of the Hamiltonian in the absence of the impurity are

$$
\psi(\rho, \varphi, z)= \begin{cases}N_{1} \mathrm{e}^{\mathrm{i} k z} \mathrm{e}^{ \pm \mathrm{i} l \phi} J_{l}\left(r_{n l} r\right), & r<R, \\ N_{1} \frac{J_{l}\left(r_{n l} R\right)}{K_{l}\left(b_{n l} R\right)} \mathrm{e}^{\mathrm{i} k z} \mathrm{e}^{ \pm \mathrm{i} l \phi} K_{l}\left(b_{n l} r\right), & r \geq R,\end{cases}
$$

where $N_{1}$ is the normalization constant,

$$
r_{n l}=\sqrt{\frac{2 m_{\mathrm{ep}}^{*} E_{n l k}}{\hbar^{2}}}
$$

and

$$
b_{n l}=\sqrt{\frac{2 m_{\mathrm{ep}}^{*}\left(V-E_{n l k}\right)}{\hbar^{2}}},
$$

where $r_{n l}$ is the $n$-th root satisfying the equation

$$
\left.r_{n l} \frac{\mathrm{d} J_{l}\left(r_{n l} r\right)}{\mathrm{d}\left(r_{n l} r\right)}\right|_{r=R}=\left.b_{n l} \frac{J_{l}\left(r_{n l} r\right)}{K_{l}\left(b_{n l} r\right)} \frac{\mathrm{d} K_{l}\left(b_{n l} r\right)}{\mathrm{d}\left(b_{n l} r\right)}\right|_{r=R} .
$$

$E_{n l k}$ is the lowest binding energy calculated by solving the transcendental equation

$$
\begin{aligned}
\sqrt{E} & J_{1}(\sqrt{E} R) K_{0}(\sqrt{(V-E)} R) \\
& =\sqrt{(V-E)} K_{1}(\sqrt{(V-E)} R) J_{0}(\sqrt{E} R) .
\end{aligned}
$$

This fixes the values of $r_{n l}$ and $b_{n l}$ for the lowest values of $E_{n l k}$ after matching the wave functions and their derivatives at boundaries of the quantum dot along with the normalization.

The electron longitudinal-optical phonon interaction is an important factor determining the physical properties of any polar crystal. The binding energies will be enhanced when a screened Coulomb potential is considered. The Aldrich-Bajaj potentials have proven to describe very well the electron-phonon interaction. We consider the effective Coulomb potential derived by Aldrich and Bajaj [22] taking into account the polaronic effects as

$$
\begin{gathered}
V_{\mathrm{AB}}(r)=-\frac{\varepsilon_{\mathrm{I}}-\varepsilon_{\mathrm{II}}}{\varepsilon_{\mathrm{I}} \varepsilon_{\mathrm{II}} R}-\frac{e^{2}}{\varepsilon r}-\frac{e^{2}}{2 \varepsilon^{\prime} r} \exp (-\beta r) \\
+\frac{e \beta}{2 \varepsilon^{\prime}} \frac{\exp (-\beta r)}{1+\alpha / 12+\alpha /(4+\alpha / 3)},
\end{gathered}
$$

where

$$
\begin{aligned}
& \beta=\left(\frac{2 m_{\mathrm{ep}}^{*} \omega}{\hbar}\right)^{1 / 2}, \\
& \alpha=\frac{e^{2} \beta}{2 \varepsilon^{\prime} \hbar \omega},
\end{aligned}
$$

and

$$
\frac{1}{\varepsilon^{\prime}}=\frac{1}{\varepsilon_{\infty}}-\frac{1}{\varepsilon_{0}},
$$

where $\omega$ is the longitudinal optical phonon frequency and $\varepsilon_{\infty}$ is the high dielectric constant of CdTe material.

If the impurity is introduced, the variation trial wave function is given by

$$
\begin{aligned}
& \psi(\rho, \varphi, z)= \\
& \begin{cases}N_{2} \mathrm{e}^{\mathrm{i} k z} \mathrm{e}^{ \pm \mathrm{i} l \phi} J_{l}\left(r_{n l} r\right) \exp (-\eta r), & r<R, \\
N_{2} \frac{J_{l}\left(r_{n l} R\right)}{K_{l}\left(b_{n l} R\right)} \mathrm{e}^{\mathrm{i} k z} \mathrm{e}^{ \pm \mathrm{i} l \phi} K_{l}\left(b_{n l} r\right) \exp (-\eta r), & r \geq R,\end{cases}
\end{aligned}
$$

where $N_{2}$ is the normalization constant and $\eta$ is the variational parameter. We calculate the ground state energy $E$ by finding out the expectation value of the energy of the Hamiltonian, Eq. (1), as

$$
\langle E\rangle=\min _{\eta} \frac{\langle\psi|H| \psi\rangle}{\langle\psi \mid \psi\rangle} \text {. }
$$

The donor binding energy $E_{\mathrm{b}}$ is calculated using the following equation:

$$
E_{\mathrm{b}}=E_{\mathrm{sub}}-\left\langle H_{\mathrm{min}}\right\rangle,
$$

where $E_{\text {sub }}$ is the lowest binding energy without the impurity. First, we concentrate on the calculation of the electronic structure of an impurity in a quantum dot by calculating their subband energy $\left(E_{\text {sub }}\right)$ and subsequently its binding energy with a variational technique. For this purpose, we use the single band effective mass approximation and expand the electron wave function in an appropriate set of orthonormal functions. Then, by using the density matrix approach [22], within a two-level system approach, the explicit expressions for the nonlinear optical properties such as the nonlinear optical absorption and the changes of refractive index are computed in a saturation limit.

\subsection{Linear and non-linear optical absorption}

The optical absorption calculations are based on the Fermi golden rule from which the total absorption coefficient is given by [23]:

$$
\alpha(\omega, I)=\alpha_{1}(\omega)+\alpha_{3}(\omega, I) .
$$

For any electronic system transitions, these calculations are imperative to compute the different optical properties. However, the dipole transitions are allowed using the selection rules $\Delta l= \pm 1$ where $l$ is the angular momentum quantum number. In addition to that the oscillator strength which is related to the dipole transition, expressed as

$$
P_{f i}=\frac{2 m_{\mathrm{ep}}^{*}}{\hbar^{2}} \Delta E_{f i}\left|M_{f i}\right|^{2},
$$

where $\Delta E_{f i}=E_{f}-E_{i}$ refers the difference of the energy between the lower and upper states. $M_{f i}=2\langle f|\boldsymbol{R}| i\rangle$ is the electric dipole moment of the transition from $i$ state to $f$ state in the quantum dot. The observation of oscillator strength is imperative especially in the study of optical properties and they are related to the electronic dipole allowed absorptions. Moreover, the outcome of the results will be viewed on the fine structure of the optical absorption. 
The optical absorption coefficient is given by

$$
\alpha_{1}(\omega)=\frac{4 \pi \alpha_{f} \sigma_{s}}{n_{r} e^{2}} \hbar \omega\left|M_{f i}\right|^{2} \delta\left(E_{f}-E_{i}-\hbar \omega\right)
$$

and

$$
\begin{gathered}
\alpha_{3}(\omega, I)=-\frac{32 \pi^{2} \alpha_{f} \sigma_{s} I}{n_{r}^{2} e^{2} \hbar \Gamma_{f f}} \hbar \omega\left|M_{f i}\right|^{2} \delta\left(E_{f}-E_{i}-\hbar \omega\right) \\
\times\left[1-\frac{\left|M_{f f}-M_{i i}\right|^{2}}{4\left|M_{f i}\right|^{2}}\right. \\
\left.\times \frac{\left(\hbar \omega-E_{f i}\right)^{2}-\left(\hbar \Gamma_{f i}\right)^{2}+2 E_{f i}\left(E_{f i}-\hbar \omega\right)}{E_{f i}^{2}+\left(\hbar \Gamma_{f i}\right)^{2}}\right],
\end{gathered}
$$

where $\sigma_{s}$ is electron density of the quantum dot, $n_{r}$ is the refractive index of the semiconductor, $\omega$ is the angular frequency of the incident photon energy, $\alpha_{f}$ is the fine structure constant and $E_{i}$ and $E_{f}$ denote the confinement energy levels for the ground and the first excited state, respectively. The above two equations are linear and third order nonlinear optical absorption coefficients. From Eq. (28) and Eq. (29), the energy-conserving delta function by the Lorentzian is given by

$$
\delta\left(E_{f}-E_{i}-\hbar \omega\right)=\lim _{\Gamma \rightarrow 0} \frac{\Gamma}{\pi\left[\left(E_{f}-E_{i}-\hbar \omega\right)^{2}+\Gamma^{2}\right]},
$$

where $\Gamma$ is the line width of the hydrogenic impurity and in our calculation we use $\Gamma=0.1 \mathrm{meV}$.

The susceptibilities are related to the refractive index changes as

$$
\frac{\Delta n(\omega)}{n_{r}}=\operatorname{Re}\left(\frac{\chi(\omega)}{2 n_{r}^{2}}\right),
$$

where $n_{r}$ is the refractive index of the material. The analytic expressions of the linear and nonlinear changes in the refractive index are given by

$$
\frac{\Delta n_{(1)}(\omega)}{n_{r}}=\frac{\sigma_{s} e^{2}}{2 \varepsilon_{r}}\left|M_{f i}\right|^{2} \frac{\hbar \omega_{f i}-\hbar \omega}{\left(E_{f i}-\hbar \omega\right)^{2}+\left(\Gamma_{f i}\right)^{2}}
$$

and

$$
\begin{aligned}
& \frac{\Delta n_{(3)}(\omega)}{n_{r}}=-\frac{\mu_{0} c I}{4 \varepsilon_{r} n_{r}^{2}} \frac{\sigma_{s} e^{4}\left|M_{f i}\right|^{4}}{\left[\left(E_{f i_{i}}-\hbar \omega\right)^{2}+\left(\Gamma_{f i}\right)^{2}\right]^{2}} \\
& \times 4\left|M_{f i}\right|^{2}\left(E_{f i}-\hbar \omega\right)-\left[\left|M_{f f}-M_{i i}\right|^{2}\right. \\
& \left.\times \frac{E_{f i}\left(E_{f i}-\hbar \omega_{f i}\right)^{2}-\left(\Gamma_{f i}\right)^{2}\left(3\left(E_{f i}-2 \hbar \omega\right)\right.}{E_{f i}^{2}+\left(\Gamma_{f i}\right)^{2}}\right] .
\end{aligned}
$$

Hence the total refractive index change is given by

$$
\frac{\Delta n(\omega)}{n_{r}}=\frac{\Delta n_{(1)}(\omega)}{n_{r}}+\frac{\Delta n_{(3)}(\omega)}{n_{r}} .
$$

\section{Results and discussion}

The ground state and low lying-excited state energies of a hydrogenic impurity located at the centre of a strained $\mathrm{Zn}_{x} \mathrm{Cd}_{1-x} \mathrm{Te} / \mathrm{ZnTe}$ quantum dot, induced by the spontaneous and piezoelectric polarizations have been calculated with various $\mathrm{Cd}$ content with and without the polaronic effect by taking into account the impurity potential using the Aldrich-Bajaj effective potential with the dielectric confinement. The effect of $z$-confinement has been calculated through a finite quantum dot model with confinement potential determined by the band offsets and strain effects. Calculations have been performed using the Bessel function as an orthonormal basis for different confinement potentials of barrier height. The atomic units have been followed in the determination of electronic charges and wave functions in which the electronic charge and the Planck constant have been assumed as unity. All the other values of $\mathrm{Cd}_{1-x} \mathrm{Zn}_{x}$ Te parameters are interpolated from the data mentioned in Table.

TABLE

Material parameters* used in the calculations.

\begin{tabular}{c|c|c}
\hline \hline Parameter & CdTe & ZnTe \\
\hline$m_{j}^{*}$ & 0.11 & 0.2 \\
$\varepsilon$ & 9.6 & 9.81 \\
$a[\mathrm{~nm}]$ & 0.6481 & 0.6466 \\
$C_{11}[\mathrm{GPa}]$ & 5.66 & 5.315 \\
$C_{12}[\mathrm{GPa}]$ & 3.96 & 1.87 \\
$E_{\mathrm{g}}^{\Gamma}[\mathrm{eV}]$ & 1.606 & 2.394 \\
\hline${ }^{*}$ Ref. $[20]$.
\end{tabular}

Figure 1 shows the variation of binding energy as a function of dot radius for various confinement potentials $(x)$ in a strained $\mathrm{Cd}_{1-x} \mathrm{Zn}_{x} \mathrm{Te} / \mathrm{ZnTe}$ quantum dot with the inclusion of polaronic effect and the inset shows the variation of barrier height with the $\mathrm{Zn}$ concentration. The hydrogenic impurity binding energy has been computed with $V_{\mathrm{AB}}$, the screened potential derived by Aldrich and Bajaj [22] taking into account the polaronic effects. The effect of attractive Coulomb potential of the impurity on the total energy is brought out here. It is observed that the binding energy increases with decreasing dot radius and it reaches the maximum value for a critical dot radius and then rapidly decreases in all the cases. It is because the contribution of confinement is dominant for smaller dot radii making the electron unbound with the spread of the wave function through the barrier [24]. The dotted points refer the results obtained by Stojanovič and Kostič [24] who calculated the hydrogenic impurity in $\mathrm{CdTe} / \mathrm{ZnTe}$ spherical quantum dot analytically. Our results are in good agreement with their analytical results. The inset shows the variation of barrier height as a function of $\mathrm{Zn}$ content in a $\mathrm{Cd}_{1-x} \mathrm{Zn}_{x} \mathrm{Te} /$ $\mathrm{ZnTe}$ quantum dot. It is found that the barrier height increases as the $\mathrm{Zn}$ concentration is increased due to the enhancement of the band gap with the concentration. Hence, the binding energy increases with the concentration of $\mathrm{Zn}$ in CdZnTe quantum dot.

Figure 2 displays the variation of binding energy as a 


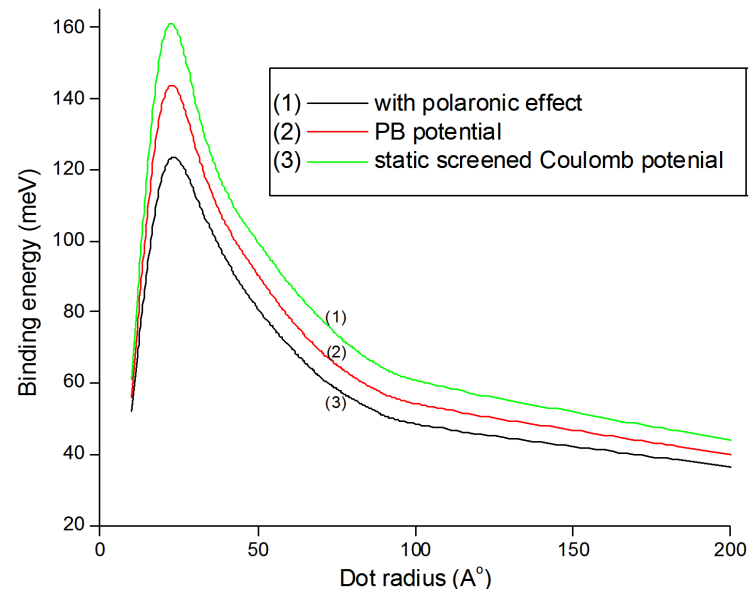

Fig. 2. Variation of binding energy as a function of dot radius in a $\mathrm{Zn}_{x} \mathrm{Cd}_{1-x} \mathrm{Te} / \mathrm{ZnTe}$ quantum dot; curve (1) represents the binding energy with the inclusion of PB potential (Eq. (19)) and the dielectric mismatch, curve (2) represents using PB potential and curve (3) is obtained using bare screened Coulomb potential.

function of dot radius in a $\mathrm{Zn}_{x} \mathrm{Cd}_{1-x} \mathrm{Te} / \mathrm{ZnTe}$ quantum dot. The curve (1) represents the binding energy with the inclusion of PB potential using Eq. (19) and the dielectric mismatch, the curve (2) represents using PB potential and the curve (3) is obtained using bare screened Coulomb potential. It is observed that the enhancement of the binding energy due to electron-phonon interaction is larger for all the dot radii but we notice that the binding energy is more influenced for smaller dot radii than the larger dot radii due to the confinement. It is important to notice that the polaronic effect is small for heterostructures with weak ionic structure whereas it is appreciable for all the polar heterostructures when the effect of polaron is included [25]. The electron-phonon interaction enhances the electron effective mass (polaron mass) and diminishes the electrostatic screening. Both the effects give rise to the enhancement of the binding energy when the effect of the polaron is included. Further we notice that the decrease of screening due to the less value of dielectric constant in $\mathrm{CdTe}$ material increases the binding energy. It is because the larger the polaron mass increases the effective Rydberg energy ultimately increases the binding energy.

Variation of absorption coefficients of a hydrogenic impurity is shown in Fig. 3 in the strained $\mathrm{Zn}_{x} \mathrm{Cd}_{1-x} \mathrm{Te} /$ ZnTe quantum dot with the radius $40 \AA$ and $80 \AA$, as a function of photon energy and $I=10 \mathrm{MW} / \mathrm{m}^{2}$ for three different $\mathrm{Zn}$ concentration and the inset shows the variation of absorption coefficients as a function of photon energy with and without the polaronic effect for a constant $\mathrm{Zn}$ concentration $(x=0.2)$. It is observed that the magnitude of the linear absorption coefficient shifts towards lower energies (red shift) as the dot radius becomes larger. It implies that size effect dominates in the low dimensional semiconductor systems. Hence there occurs a

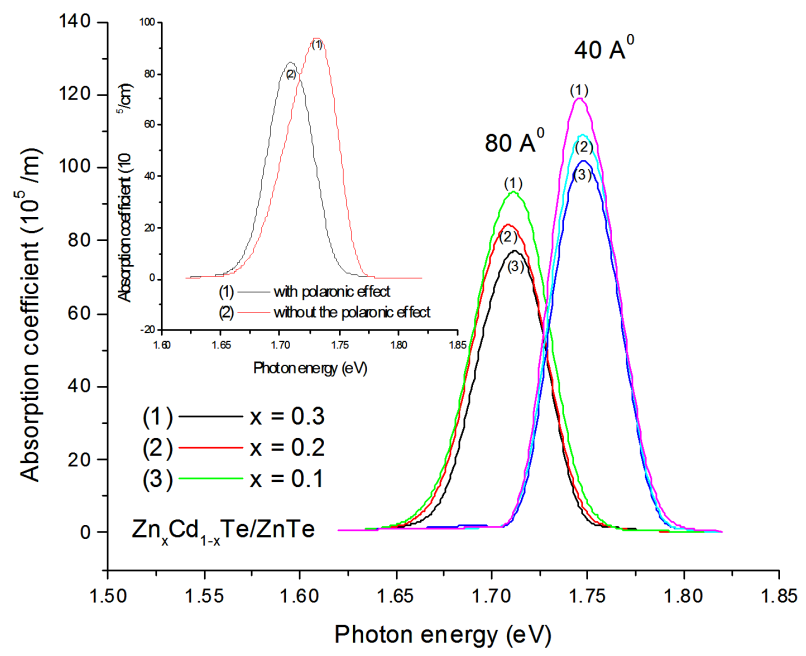

Fig. 3. Variation of absorption coefficients of a hydrogenic impurity in the strained $\mathrm{Zn}_{x} \mathrm{Cd}_{1-x} \mathrm{Te} / \mathrm{ZnTe}$ quantum dot with the radius $40 \AA$ and $80 \AA$, as a function of photon energy and $I=10 \mathrm{MW} / \mathrm{m}^{2}$ for three different $\mathrm{Zn}$ concentration; the inset shows the variation of absorption coefficients as a function of photon energy with and without the polaronic effect for a constant $\mathrm{Zn}$ concentration $(x=0.2)$.

blue shift when the dot radius is increased. Also, we notice that the absorption coefficients of smaller dot radii are stronger than that of the larger dot radii. It is because the increase of the Coulomb interaction energy leads to the increase of the energy difference between the initial and final states. Moreover, it is obvious that the size of the quantum dot depends on the transition matrix element, the electron density and ultimately on the absorption coefficients. Moreover, we notice that the binding energy is more for when the polaronic effect is included for all the dot radii due to the enhancement of hydrogen binding energy when the Hamiltonian is included with the polaronic effect. Hence it is concluded that intensity dependent nonlinear absorption coefficients near the resonant frequencies are important and it should be taken into account in studying the optical properties of hydrogenic impurity in the low dimensional heterosystem.

However, the contribution from the nonlinear optical absorption coefficient should be considered provided the optical intensity is very strong. The intensity of the total absorption spectra increases for the transition between higher levels due to the increase of electronic dipolar matrix element. When we include the effect of phonon the optical absorption peak increases remarkably due to the increase of electronic dipolar matrix element with the strong confinement potential. The phonons in strong ionic crystals involve the relative motion of positive and negative ions occurring polarization and has a strong interaction with electromagnetic waves. Thus the LO phonon has a remarkable influence on the optical properties of polar crystals. Moreover, phonon has an important effect to the electron transition between the inter- 


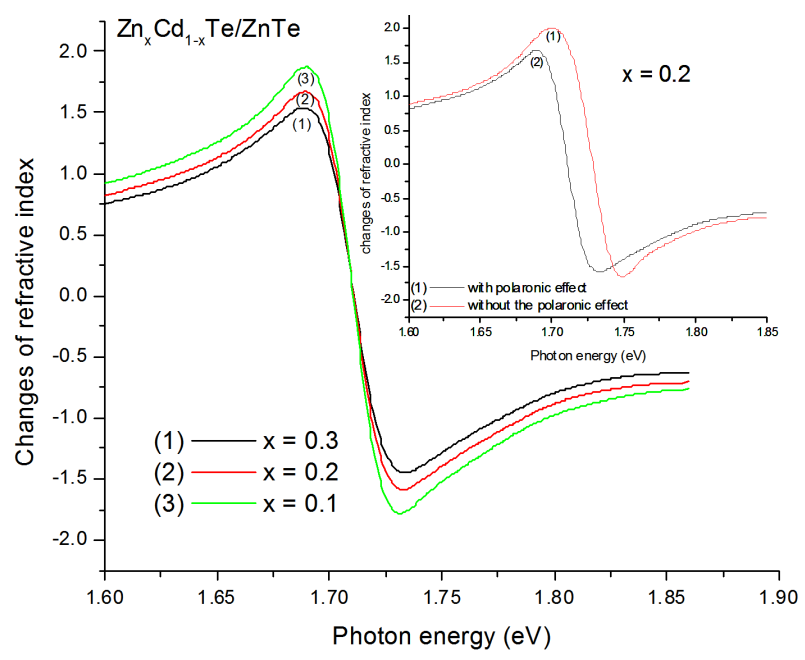

Fig. 4. Variation of changes of refractive index for a hydrogenic impurity in the strained $\mathrm{Zn}_{x} \mathrm{Cd}_{1-x} \mathrm{Te} / \mathrm{ZnTe}$ quantum dot with the radius $40 \AA$, as a function of photon energy and $I=10 \mathrm{MW} / \mathrm{m}^{2}$ for three different $\mathrm{Zn}$ concentration; the inset shows the changes of refractive index as a function of photon energy with and without the polaronic effect.

subband when the system is irradiated with the photon. Hence, the total optical absorption magnitude increases by a factor of $2-3$ with the inclusion of the electronphonon interaction taken into account [26].

Figure 4 shows the variation of changes of refractive index for a hydrogenic impurity in the strained $\mathrm{Zn}_{x} \mathrm{Cd}_{1-x} \mathrm{Te} / \mathrm{ZnTe}$ quantum dot with the radius $40 \AA$, as a function of photon energy and $I=10 \mathrm{MW} / \mathrm{m}^{2}$ for three different $\mathrm{Zn}$ concentration and the inset shows the changes of refractive index as a function of photon energy with and without the polaronic effect. This figure has been drawn with the combining effects of two components of refractive index, namely, $\frac{\Delta n_{(1)}(\omega)}{n_{r}}$ and $\frac{\Delta n_{(3)}(\omega)}{n_{r}}$ as a function of incident energy with and without the polaronic effect with the constant incident optical intensity. It is observed that as the $\mathrm{Zn}$ incorporation increases, the total refractive index changes shift towards the higher values and the magnitude of total refractive index increases. This is because the increase in hydrogenic impurity binding energy occurs with the Zn-composition. Further, it is noticed that the changes of refractive index moves with the higher energy when the polaronic effect is included, this is because the enhancement of the binding energy occurs due to the inclusion of polaronic effect. Also, it is noticed from Eq. (32) and Eq. (33) that the linear relative change in refractive index does not depend on photon intensity but the third order relative change in refractive index changes with photon intensity and it varies quadratically with the matrix element of the electric dipole moment of the transition. Thus, the nonlinear term must be considered when calculating the refractive index changes of low dimensional semiconduc- tor systems in which the incident light propagates along the $z$-axis [27]. Thus, the nonlinear term must be considered when calculating the refractive index changes in low dimensional semiconductor systems.

\section{Conclusion}

The ground state binding energy of a hydrogenic donor is investigated in a polar quantum dot nanostructure. We have studied the optical properties of hydrogenic impurity in a strained $\mathrm{Zn}_{x} \mathrm{Cd}_{1-x} \mathrm{Te} / \mathrm{ZnTe}$ quantum dot with and without the inclusion phonon assuming a spherically confinement potential for various $\mathrm{Zn}$ concentration. Variation of donor binding energy as a function of dot radius for various $\mathrm{Zn}$ content has been performed using single band effective mass approximation. Calculations have been obtained using the Bessel function as an orthonormal basis for different confinement potentials of barrier height considering the internal electric field induced by the spontaneous and piezoelectric polarizations. The magnitude of the absorption coefficients and the refractive index changes will increase for transitions between higher levels with the inclusion of phonon effect. Our results are presented for various dot radii, $\mathrm{Zn}$ content and confining potentials. It is investigated that the potential with the inclusion of phonon will make the hydrogenic binding energies more than the obtained results using a Coulomb potential screened by a bare static dielectric constant and the optical properties of hydrogenic impurity in a quantum dot are strongly affected by the confining potential, dot radii and the $\mathrm{Zn}$ composition. We hope that our results would explore new findings in experimental sides on electro-optical devices.

\section{References}

[1] L.M. Liu, J.J. Li, G.G. Xiong, Physica E 25, 466 (2005).

[2] Zh.Q. Gui, G.G. Xiong, F. Gao, Microelectron. J. 38, 447 (2007).

[3] B. Li, K.X. Guo, Ch.J. Zhang, Y.B. Zheng, Phys. Lett. A 367, 493 (2007).

[4] G. Wang, K. Guo, Physica E 28, 14 (2005).

[5] W. Xie, Phys. Lett. A 372, 5498 (2008).

[6] M. Sahin, J. Appl. Phys. 106, 063710 (2009).

[7] G. Rezaei, M.R.K. Vahdani, B. Vaseghi, Curr. Appl. Phys. 11, 176 (2011).

[8] H.S. Lee, H.L. Park, T.W. Kim, Appl. Phys. Lett. 92, 052108 (2008).

[9] J.M. Nieto, F. Comas, Physica B Condens. Matter 388, 153 (2007).

[10] M. Goryca, Proc. SPIE 7600, 76001N (2010).

[11] T. Kazimierczuk, J. Suffczynski, A. Golnik, J.A. Gaj, P. Kossacki, P. Wojnor, Phys. Rev. B 79, 153301 (2009).

[12] T. Kazimierczuk, A. Golnik, M. Goryca, P. Wojnor, J.A. Gaj, P. Kossacki, Acta Phys. Pol. A 116, 882 (2009). 
[13] J.H. You, J.T. Woo, D.U. Lee, T.W. Kim, K.H. Yoo, H.L. Park, Opt. Quant. Electron. 41, 559 (2009).

[14] A.L. Vartanian, L.A. Vardanyan, E.M. Kazaryan, Phys. Lett. A 360, 649 (2007).

[15] Y.F. Huangfu, Z.W. Yan, Physica E 40, 2982 (2008).

[16] Z. Samak, B. Saqqa, An-Najah Univ. J. Res. (N. Sc.)23, 15 (2009).

[17] A.L. Vartanian, M.A. Yeranosian, A.A. Kirakosyan, J. Contemp. Phys. (Armen. Acad. Sci.) 42, 230 (2007).

[18] Wu Yun-Feng, Liang Xi-Xia, K.K. Baja, Chin. Phys. 14, 2314 (2005).

[19] J.J. Shi, Z.Z. Gan, J. Appl. Phys. 94, 407 (2003).

[20] S. Adachi, Properties of Semiconductor Alloys: Group IV, III-V and II-VI Semiconductors, Wiley, Oxford 2009.
[21] C.-H. Liu, B.-R. Xu, Phys. Lett. A 372, 888 (2008).

[22] C. Aldrich, K.K. Bajaj, Solid State Commun. 22, 157 (1977).

[23] J.S. de Sousa, J.P. Leburton, V.N. Freire, E.F. da Silva, Phys. Rev. B 72, 155438 (2005).

[24] D. Stojanovic, R. Kostic, Acta Phys. Pol. 120, 234 (2011).

[25] R.A. Escorcia, C. Riva, I.D. Mikhailov, Solid State Commun. 131, 365 (2004).

[26] Chao-Jin Zhang, Kang-Xian Guo, Physica E 39, 103 (2007).

[27] Wenfang Xie, J. Phys., Condens. Matter 21, 115802 (2009). 